\title{
Improving documentation and junior doctor confidence on COVID-19 ward rounds using a ward round pro forma
}

\author{
Authors: Niall Brown, ${ }^{\mathrm{A}}$ Joseph Horne ${ }^{\mathrm{A}}$ and Andrew Low ${ }^{\mathrm{A}}$
}

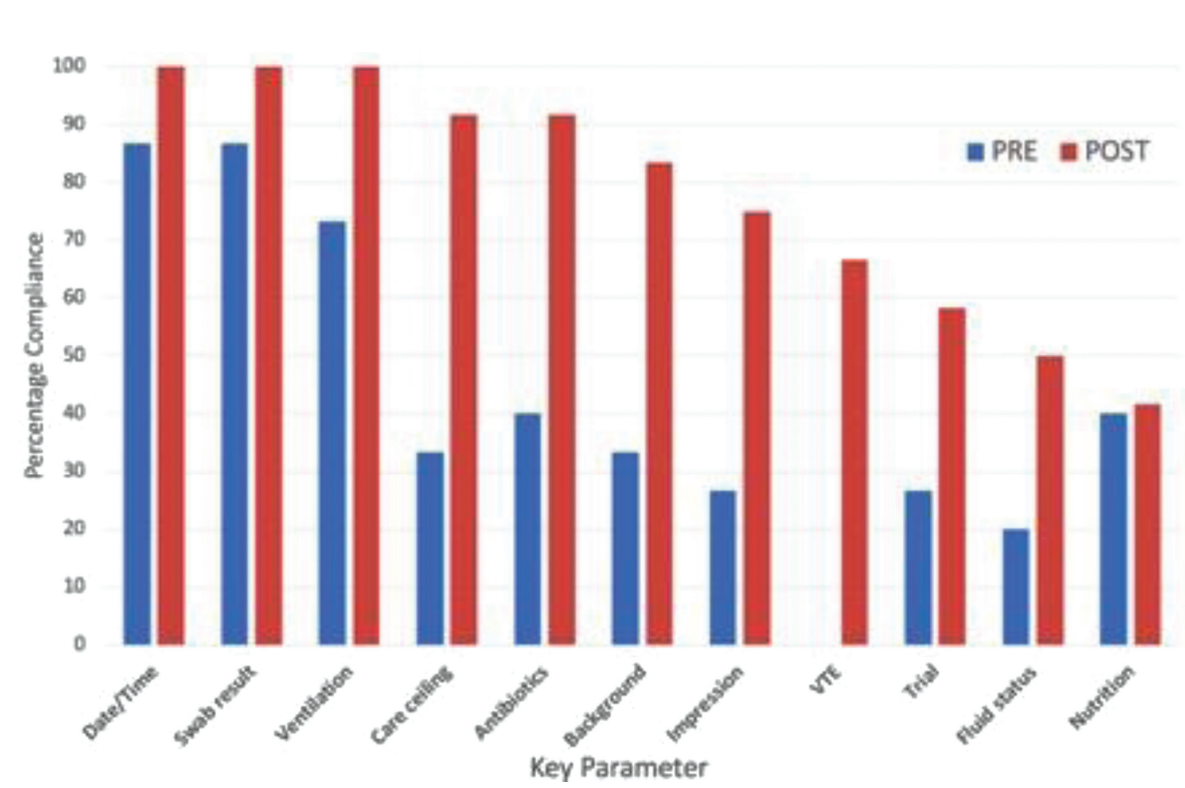

Fig 1. Documentation rates of key parameters pre- and post-implementation.

\section{Introduction}

This project developed and analysed the impact of a ward round pro forma across three wards of a large tertiary hospital containing positive or high-risk COVID-19 patients. Templates and checklists have improved patient outcomes in medical, surgical and high-care settings. ${ }^{1,2}$

The daily consultant-led ward round entry serves as the primary communication between the multidisciplinary team (MDT) and acts as the medicolegal record. There are barriers in junior doctors scribing complete ward round entries using blank sheets. As a result, pertinent information might be missed and patient care may suffer due to incomplete records.

The primary aim was to increase the amount of information documented during ward rounds $(20 \%$ increase in number of parameters) and increase junior doctor confidence in documenting (1 Likert point). The second aim was to make it easier for the MDT to find relevant information (1 Likert point). Aims were to be achieved after 2 weeks of use, aiming for $75 \%$ uptake.

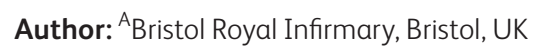

\section{Methods}

One closed-loop plan, do, study, act (PDSA) cycle was performed. Respiratory consultants and registrars determined 11 key parameters that should be documented daily for COVID-19 patients.

Baseline objective data collection analysed all high-care ward round entries $(n=15)$ on 1 day, recording key parameters as present or absent. Baseline subjective opinions on documentation were collected with an MDT questionnaire.

The ward round pro forma was trialled for 2 weeks for all COVID-19 ward inpatients; repeated subjective and objective analysis was performed. All $(\mathrm{n}=12)$ inpatient notes on the COVID-19 high-care ward were analysed.

\section{Results and discussion}

Compliance rate for pro forma use was $100 \%$. The mean number of key parameters documented on each ward round entry increased from 5 to 9 , representing an 80\% increase. Eleven (100\%) of the key parameters showed an increased completion rate (Fig 1). Junior doctor confidence in documenting increased 1 point on the Likert scale, corresponding to a change from 'average' to 


\section{5}

= PRE

- POST

章

Fig 2. Multidisciplinary team opinions on COVID-19 documentation pre- and post-implementation.

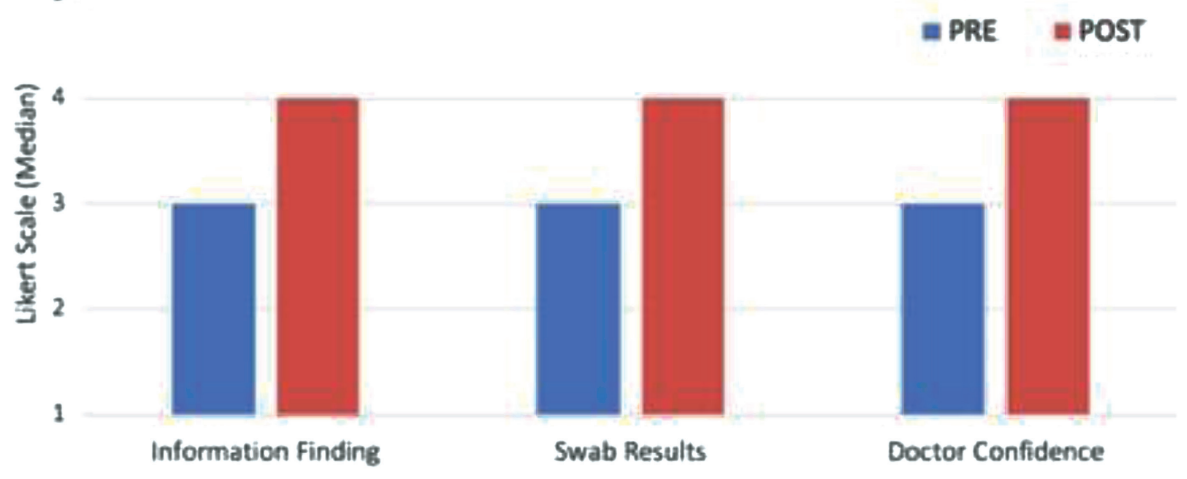

'confident' (Fig 2). Ninety-seven per cent $(n=31)$ of respondents reported that the pro forma saved time. Information finding and swab result tracking both increased by a median of 1 point on the Likert scale, corresponding to an improvement from 'average' to 'easy' (Fig 2).

The pro forma itself likely acts as a prompt. Documentation of venous thromboembolism (VTE) prophylaxis increased from $0 \%$ to $67 \%$, giving evidence of consideration according to the prothrombotic nature of the disease. ${ }^{3}$ Antibiotic documentation increased by $52 \%$ suggesting that the indication was reviewed and altered accordingly. From a medicolegal perspective, documentation of basic information (ie date and time) improved by $13 \%$. COVID-19 patients can deteriorate quickly; it is essential that their ceiling of care has been reviewed by consultant. This project shows not only a $60 \%$ increase in ceiling of care documentation, but also improved ease in accessing such information.

The principle pro forma drawbacks were space limitation and low patient numbers limiting firm statistical conclusions.

\section{Conclusion}

The pro forma designed and implemented in this project is a simple and acceptable intervention to improve documentation, giving junior doctors more confidence in their role. Further PDSA cycles are limited by reducing COVID-19 admissions.

\section{Conflicts of interest}

None declared.

\section{References}

1 Gilliland N, Catherwood N, Chen S et al. Ward round template: enhancing patient safety on ward rounds. BMJ Open Quality 2018;7:e00170.

2 Sparkes D, Rylah B. The World Health Organization surgical safety checklist. Br J Hosp Med (Lond) 2010;71:276-80.

3 Kollias A, Kyriakoulis K, Dimakakos E et al. Thromboembolic risk and anticoagulant therapy in COVID-19 patients: emerging evidence and call for action. Br J Haematol 2020;189:846-7. 